\title{
THE FORMATION, STRUCTURE AND CHANGES IN FILAMENTS IN ACTIVE REGIONS
}

\author{
SARA F. SMITH \\ (Lockheed Solar Observatory, Calif., U.S.A.)
}

During periods of good image quality the large-scale films of the Lockheed Solar Observatory obtained during 1966 and 1967 have proven to be especially useful for studying filaments and their relationship to the $\mathrm{H} \alpha$ fine structure observed in active regions. Structures with dimensions on the order of $1 \mathrm{sec}$ of arc can frequently be resolved, as illustrated in Figure 1. The 'solar vortices' are clearly resolved into numerous individual fibrils. Bright $\mathrm{H} \alpha$ plage is resolved into a fine granular structure resembling the solar granulation. Filaments are usually seen as irregular dense

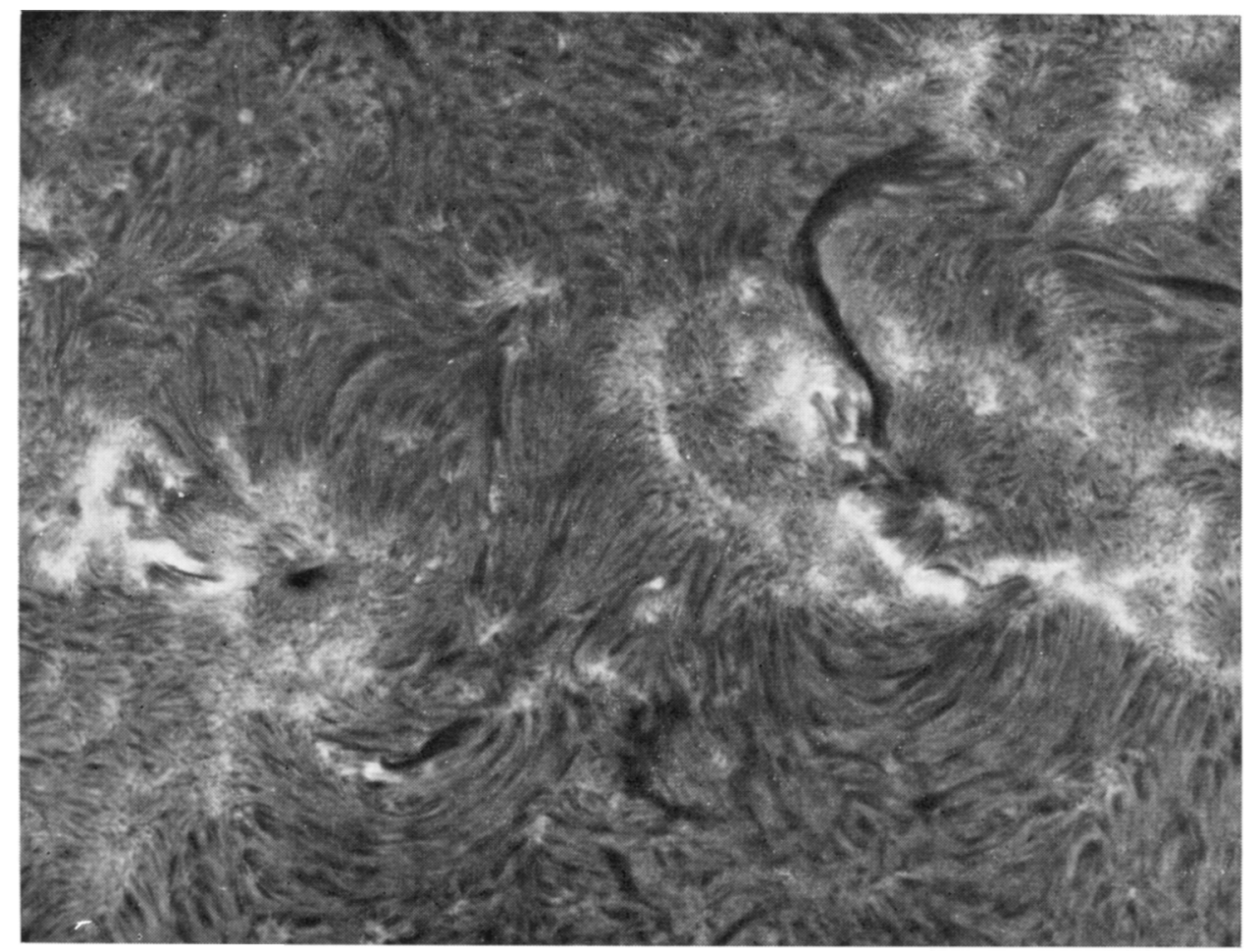

Fig. 1. H $\alpha$ fine structure in an active region, August 1, 1966. Bright plage is often resolved into fine mottles. The fine thread-like structures or 'fibrils' are shortest at the plage boundary.

Kiepenheuer (ed.), Structure and Development of Solar Active Regions, 267-279. (c) I.A.U. 
collections of material which are occasionally resolved into finer striations which run parallel to the path of the filaments.

Before discussing observed relationships between filaments and plage granules, fibrils and sunspots, it seems preferable to reiterate a few aspects of active regions that are already well known. It has been stated by Tsap (1963) that the structure and geometry of active regions are controlled by the configuration and strength of the magnetic fields of regions. Our interpretation of the observations is similar. We concur with other investigators in saying that fibrils appear to be a manifestation of the direction of the lines of force in the chromosphere.

From the work of Avignon et al. (1964), Howard (1959), Howard and Harvey (1964), Martres et al. (1966), and ourselves (1967), it is well established that filaments both inside and outside of active regions are invariably observed at the boundary between positive and negative polarities in magnetic fields. An example is presented in the right corner of Figure 2. In the lower half of Figure 2 is a fine scan magnetogram of the longitudinal component of a region recorded with a 10 sec-of-arc resolution at the Mt. Wilson Observatory. Positive polarity is represented by solid lines and negative polarity by dashed lines. In the upper half of Figure 2 is a center $\mathrm{H} \alpha$ photograph taken at Lockheed on the same day, June 2, 1967. Although the difference in resolution between the magnetogram and filtergram is a factor of 10 , the filament very nearly follows the border between positive and negative polarity. We often refer to this boundary between polarities as the line of $O$ longitudinal field or, sometimes, as the 'neutral' line. Lines of $O$ longitudinal field exist in all active regions observed near the center of the disk. They may also exist between two active regions or between remnants of old active regions. Correspondingly, filaments may be observed along any of these locations.

It has also been mentioned by Martres et al. (1966) that the boundary of polarity change may also be crossed by 'filamenteuses' or fibrils extending between adjacent plage of opposite polarity. These authors have also suggested that such fibrils may be related to the evolution of filaments. Examples of fibrils crossing the boundary between areas of opposite polarity are also shown in Figure 2. At other locations the boundary between opposing polarity is coincident with the path of a filament. In general, filaments and fibrils are not observed at the same location on a line of $O$ longitudinal field (Smith and Ramsey, 1966).

Since filaments very closely follow the boundary of a polarity change, we previously thought, along with other investigators, that the path of a filament ran perpendicular to a general pattern of lines of force in the chromosphere and low corona. In fact, this view of filaments has been so commonly held that a number of theoretical models of

FIG. 2. H $\alpha$ filtergram and Mt. Wilson fine scan magnetogram, June 2, 1967. The fibrils in plage near the large spot join plage of opposite polarity. 

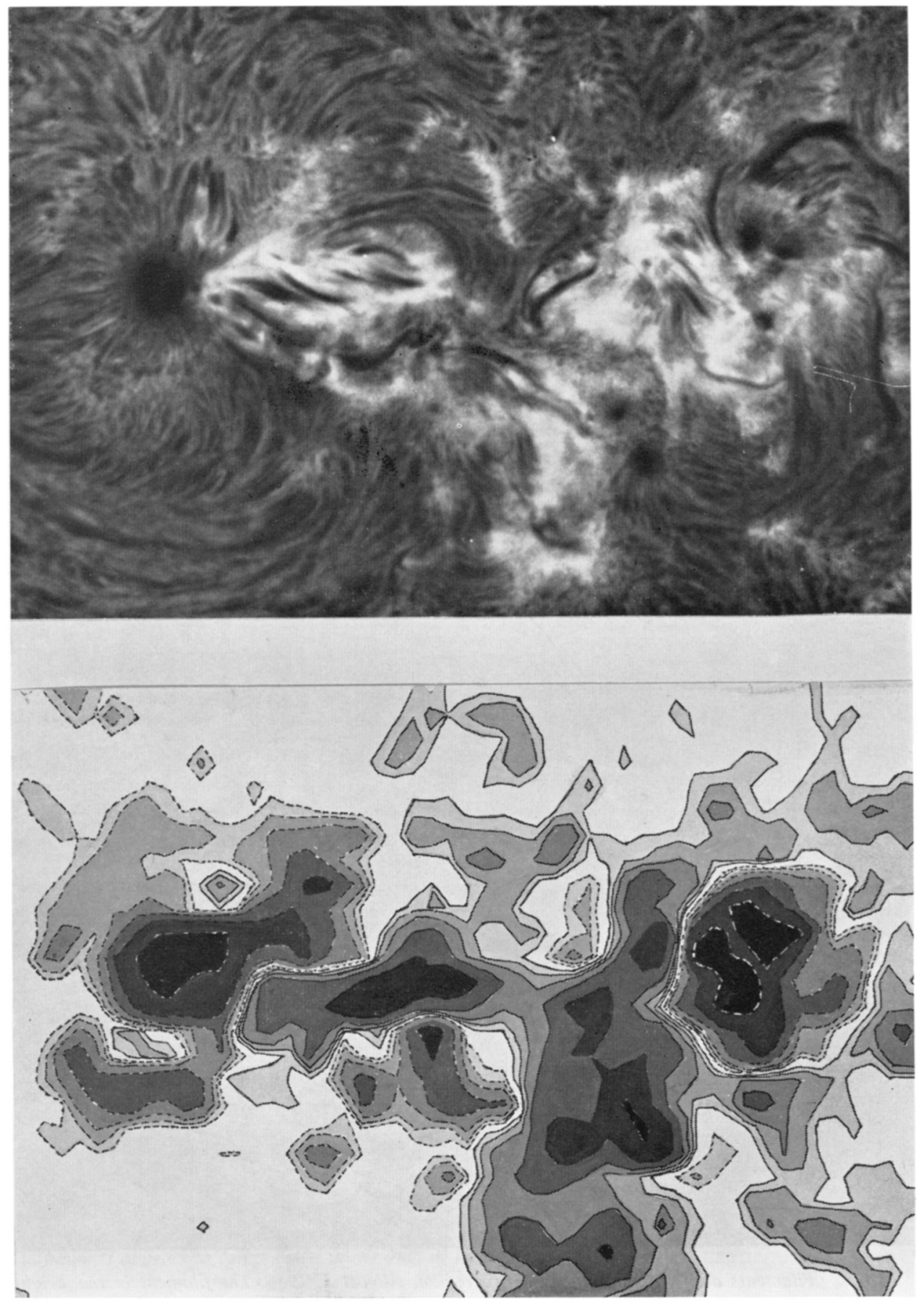
filaments have been put forth which require a deformation, sag, or distortion of the lines of force in order to make it theoretically possible for filaments to exist along lines of $O$ longitudinal field.

After studying the relationship of filaments to fibrils, plage granules, and sunspots, however, the $\mathrm{H} \alpha$ observations seem to be more consistent with the view that filaments follow a restricted set of lines of force in magnetic regions.

Figures 1, 3, and 4 illustrate the observed relationship of filaments and fibrils in active regions. When viewed near the center of the Sun, the fibrils immediately adjacent to a filament are aligned parallel or very nearly parallel to the path of the filament. A few seconds of arc away from the edge of the filament the fibrils may appear to bend away from the path of the filament, as seen in Figure 3. At the ends of filaments, two conditions have been observed. In some cases, the fibrils remain aligned parallel to the path of the filament. In other cases, a filament appears to terminate just before fibrils which appear to run nearly perpendicular to the end of the filament path. Very close inspection of these cases under conditions of good image

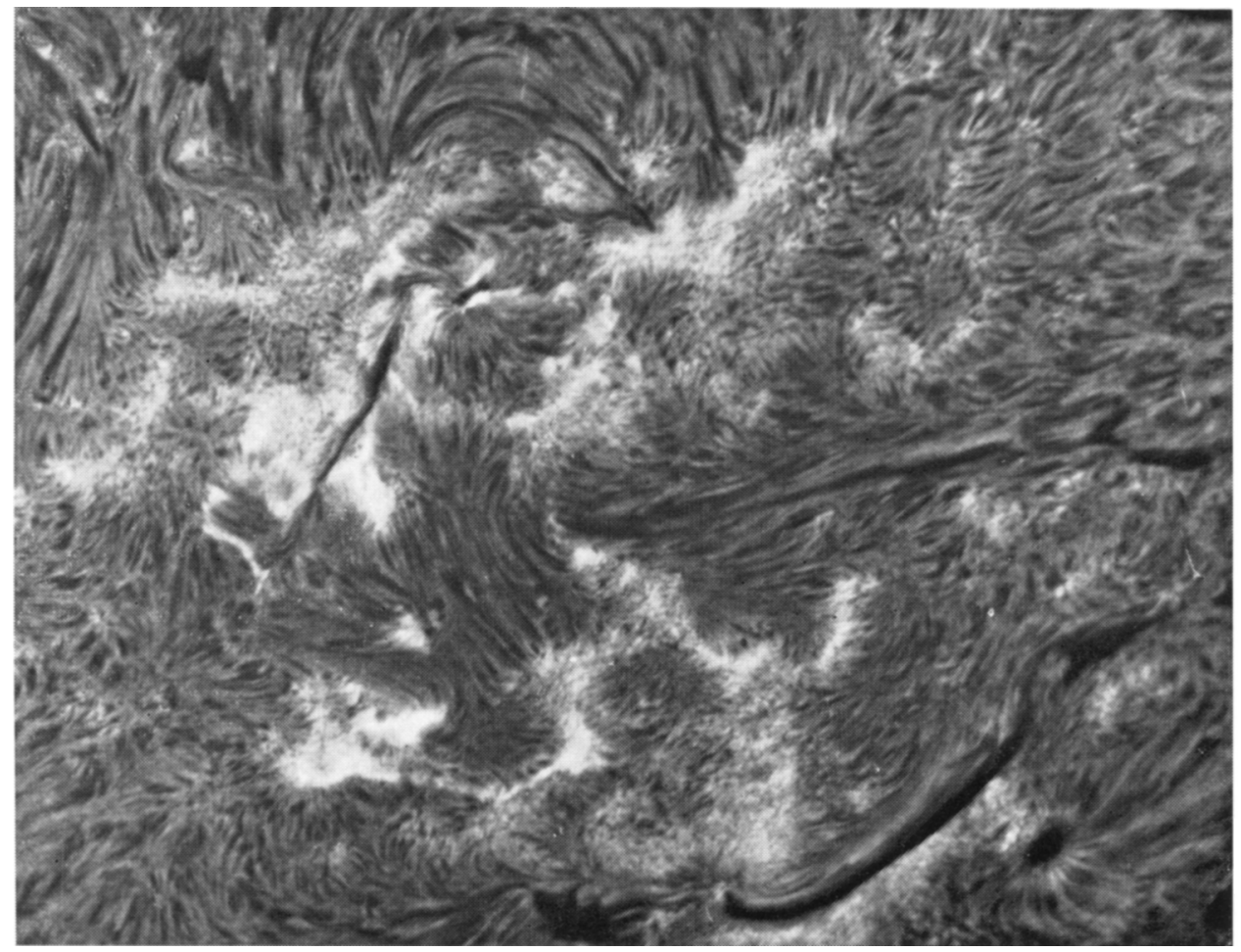

FIG. 3. Filaments and structures in an active region, August 3, 1966. The filament in the bright plage is bordered by plage granules rather than by fibrils. 


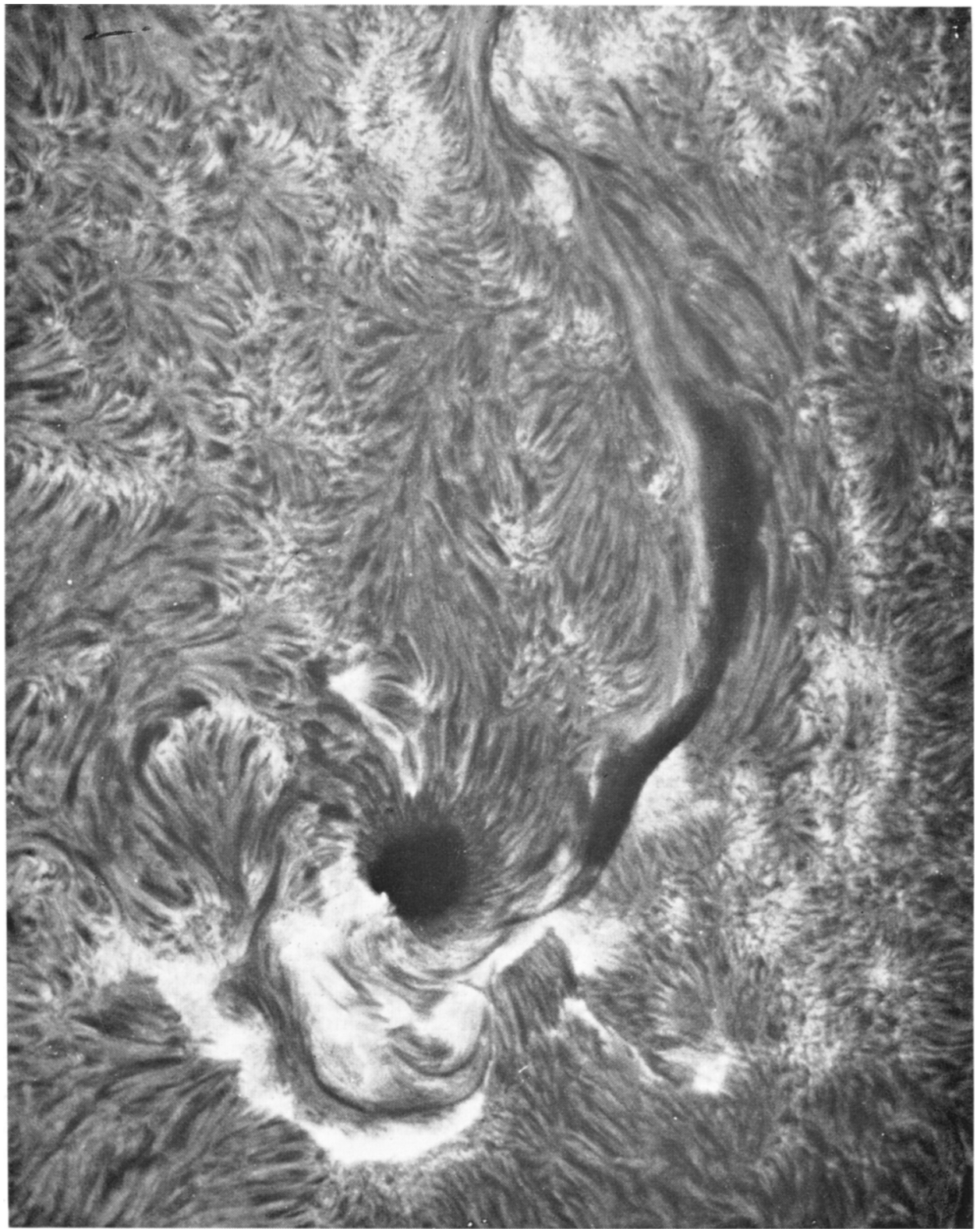

FIG. 4. Filament and adjacent fibrils in an active region, September 17, 1966. Internal structure in the filament appears to run parallel to the path of the filament. 
quality, shows that the end of the filament, in at least some cases, abruptly turns in the direction of the neutral-line fibrils. Note ends of filaments in Figure 1, 3, and 4. Generally, when atmospheric image quality is good all points along the path of filaments appear to be aligned in the same direction as adjacent fibrils. However, occasional exceptions have been observed attributable to differences in the height of filament and apparently adjacent fibrils or to effects of transient phenomena.

At some locations in active regions filaments are bordered by bright plage rather than by fibrils. The fibril structure also shows a definite patterned relationship to the plage granules, as well as to filaments. We have previously shown that the length of fibrils, except for those extending between areas of opposite polarity, show a general inverse relationship to the strength of the longitudinal component of the magnetic field (Smith and Ramsey, 1967). Except at locations where the longitudinal magnetic field changes polarity, the fibrils near plage are very short and those at the outer edge of a region tend to be longer (Figure 1). The geometry and sizes of the fibrils and plage granules are such as to suggest that the plage granules may be fibrils which extend approximately radially out from the chromosphere. Projection of the largescale time-lapse films reveals continuous motion in the plage granules and fibrils, as well as in filaments. The observed motion is also consistent with the view that plage granules could be a cross-section view of bundles of fibrils which extend nearly radially out from the chromosphere although the time scale of the motion in plage granules and fibrils appears to differ. When viewed in the center of the disk, the motion in plage granules resembles the motion of the solar granulation. There is a relatively rapid appearance and disappearance of individual granules with corresponding brightness changes suggesting a strong component of motion in the line of sight. The motion in fibrils is invariably confined along the path of the fibril. In the non-activated or usual state, the motion in active-region filaments is also along the path of the filament. The motion in filaments is thus restricted to the same directions as the motion in fibrils immediately adjacent to filaments.

Figure 5 shows the same filament depicted in Figure 4 as a path which is favorable for the flow of material. During 7 days of observation, distinct flow of emission was observed 15 times along this filament. The flow along filaments occurs both in absorption and emission. The flow of material along filaments is occasionally seen in 10 or 15 -sec interval time-lapse films projected at 16-24 frames per second. Experiments in projecting these films at rates in the range of 60-500 frames per second, however, reveal faint and subtle flow not readily detectable at slower projection rates. At projection rates around 100 frames per second and faster (1000 times real time), a continuous flow of material may be observed along the path of most filaments. Although not always readily visible, such flow, of course, must exist if filaments and prominences are still to be regarded as the same features.

From prominence observations, it has been demonstrated that prominence material may rain into sunspots. However, narrow-band $\mathrm{H} \alpha$ observations seldom reveal a 


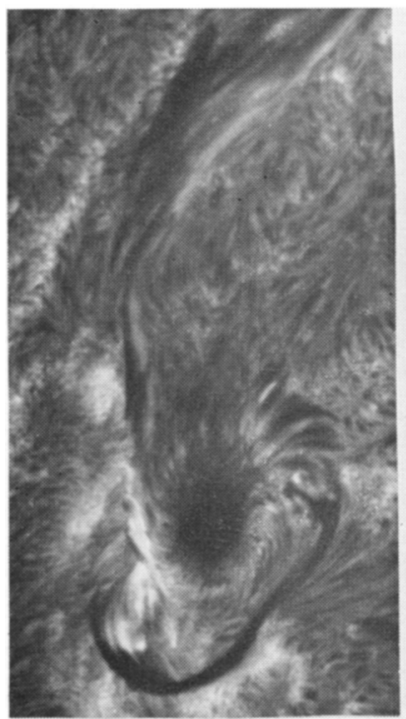

1814

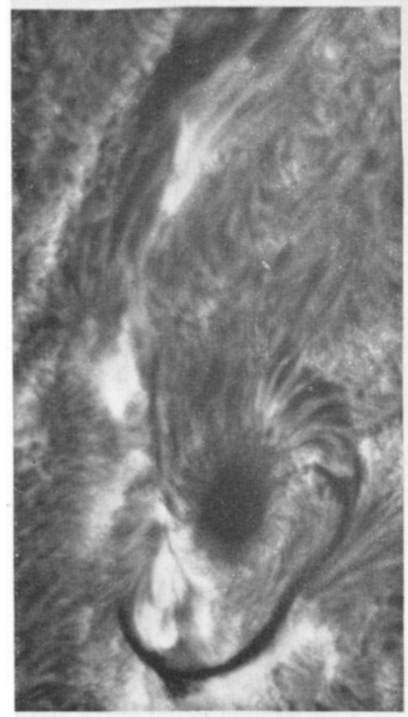

1835

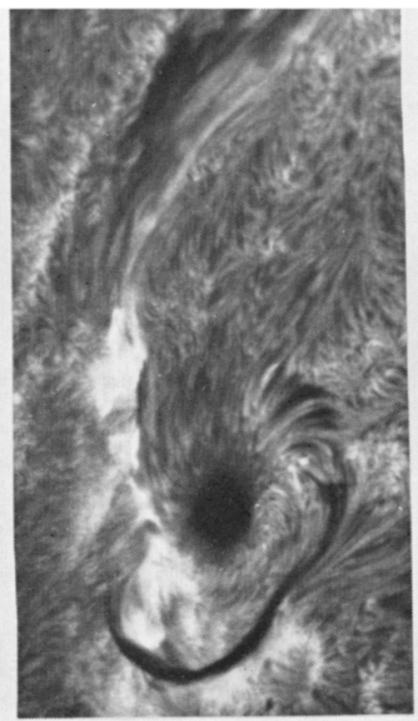

1822

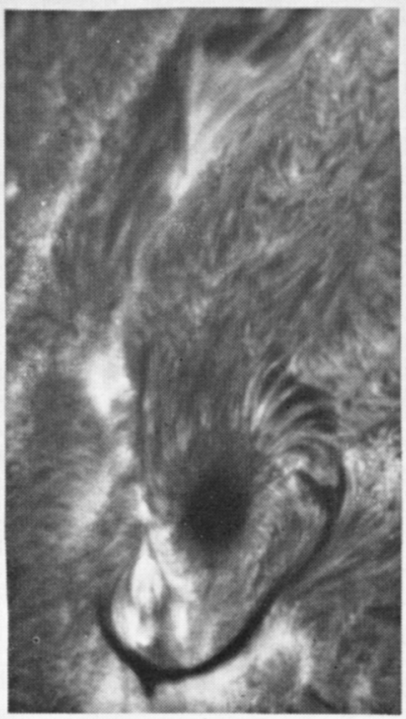

1839

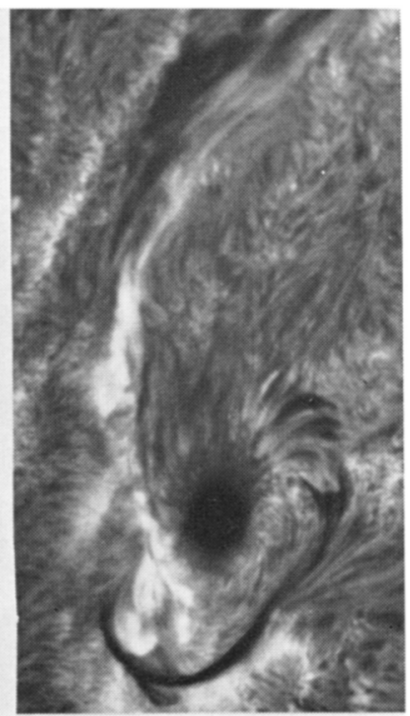

1828

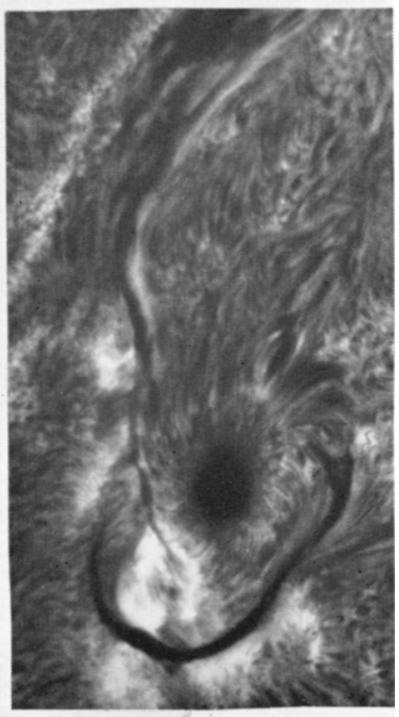

2127

Fig. 5. Flow of emission along a filament, September 15, 1966. This flow of emission is initiated by a subflare. 


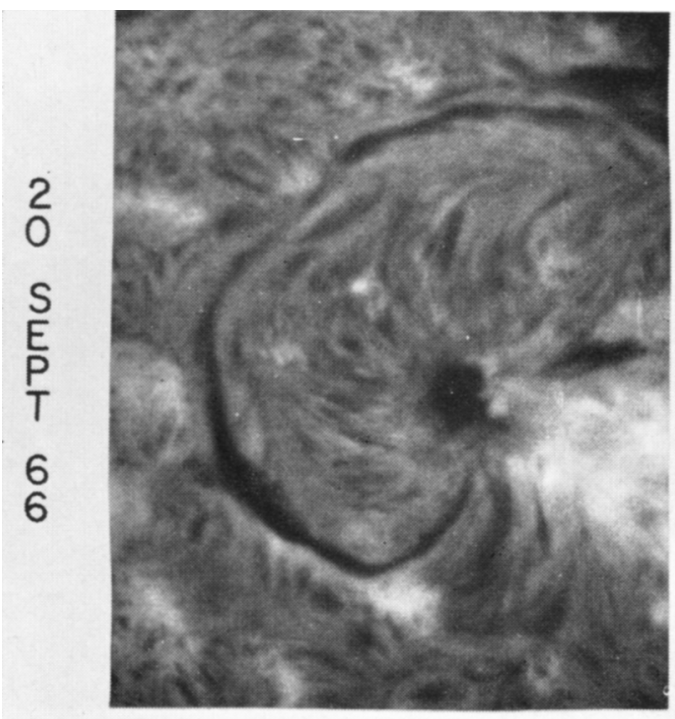

$2146: 30$

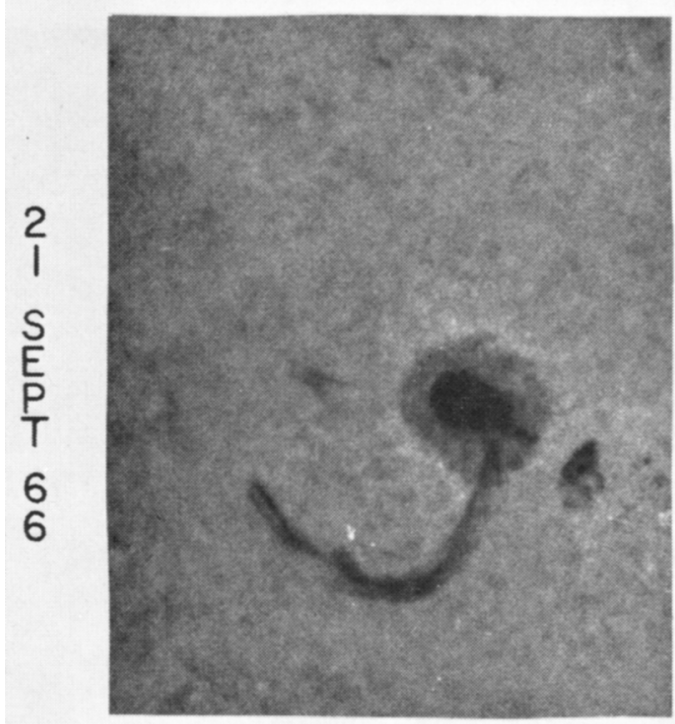

$1933: 30$

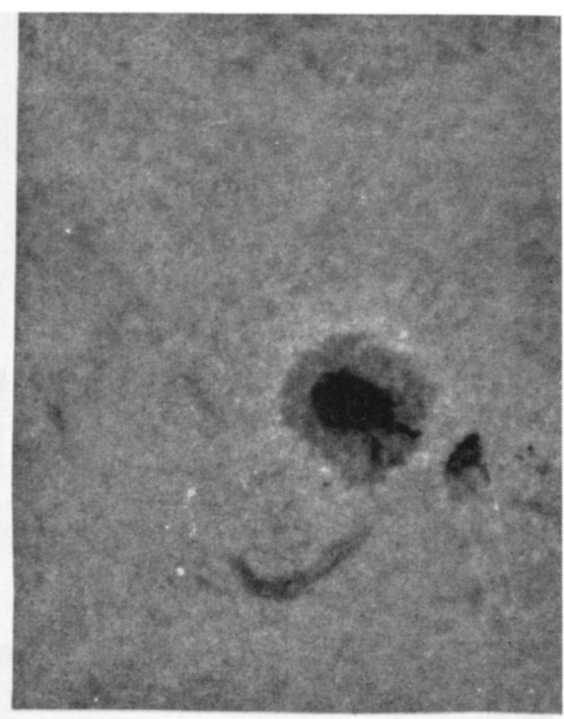

$1928: 10$

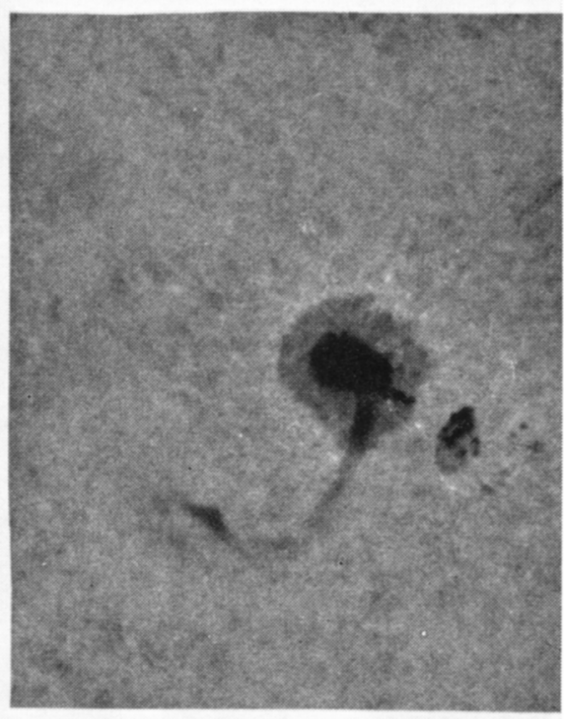

$1939: 10$

FIG. 6. Flow of filament material into a sunspot, September 20 and 21, 1966. The flow of material into the sunspot originates in the flament in the first frame. 
connection between flow of material in filaments and the flow of material into sunspots. That this connection exists can be shown unambiguously by combining narrowband and broad-band $\mathrm{H} \alpha$ observations, as presented in Figure 6. On September 21, we change from observing a $\frac{1}{2} \AA$ bandpass in the center of $\mathrm{H} \alpha$ to simultaneously observing two $\frac{1}{2} \AA$ wide transmission bands centered approximately $1 \AA$ into each wing from line center. In the narrow-band $\left(\frac{1}{2} \AA\right)$ observations on September 20 , the filament appears to terminate an appreciable distance from the sunspot. In the broader band observations on September 21, an occasional flow of material continues from the location where the filament appears to terminate in centre $\mathrm{H} \alpha$ and flows directly into the sunspot umbra. This flow of material into the umbra of this spot was observed 18 times on 4 successive days of similar observations of this region. Additional observations are needed to demonstrate the frequency of occurrence of visible flow of material from filaments to sunspot umbra. We conclude that flow from filaments into spots is probably the disk counterpart of prominence flow into sunspots which has previously been deduced by other investigators from observations at and near the limb.

A final demonstration of filaments as being preferred paths for material flow in active regions is shown in Figures 7 and 8, which illustrates an observed process of filament formation. The first stage of filament formation is the alignment of fibrils as in the left of Figure 7. This fibril alignment provides a path for material flow over long distances relative to the dimensions of individual fibrils. The flow is represented in the center photograph by two dark strands which follow the general direction of the fibrils. The photo on the right in Figure 7 shows the filament after the strands of flowing material have increased and gradually merged together in the process of filament formation. The flow of material continues throughout the observed life of the filaments.

Figure 8 illustrates the formation of a section of a filament in plage where only very short fibrils or plage granules are observed. The observed process of formation, however, is similar to the situations in which long fibrils are observed. On April 30, 1967 only a few short-lived strands of absorption appeared at the location destined for the new filament section. On May 1, 1967, strands of absorption more frequently appeared, clearly travelled across a single path almost parallel to a nearby section of filament, and then disappeared. Initially most of the strands of absorption were shortlived, lasting only a few minutes. Some of the longer-lived and darker strands appeared to be ejected from small subflares originating near the sunspot in Figure 8. During the observing period on May 2, these moving strands of absorption became a new filament section with the flow of material continuing along the same path, as defined by the strands of absorption on the previous day.

Each of these observations presented, the alignment of fibrils and filaments, the direction of flow of material along fibrils and filaments, the flow of filament material into a sunspot, and the formation of filaments only along paths of aligned fibrils, 

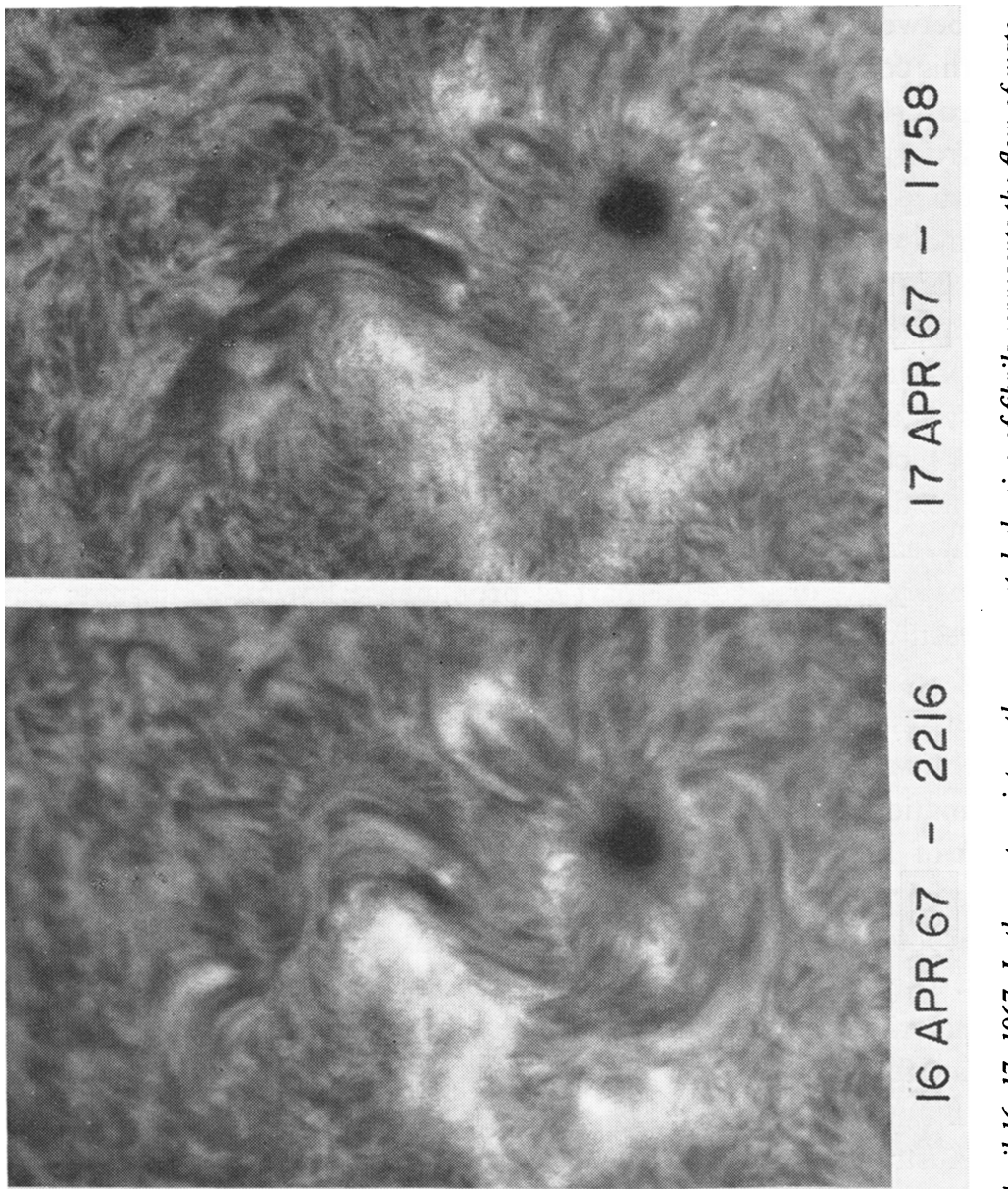

ปิ

1
0
$\frac{1}{4}$
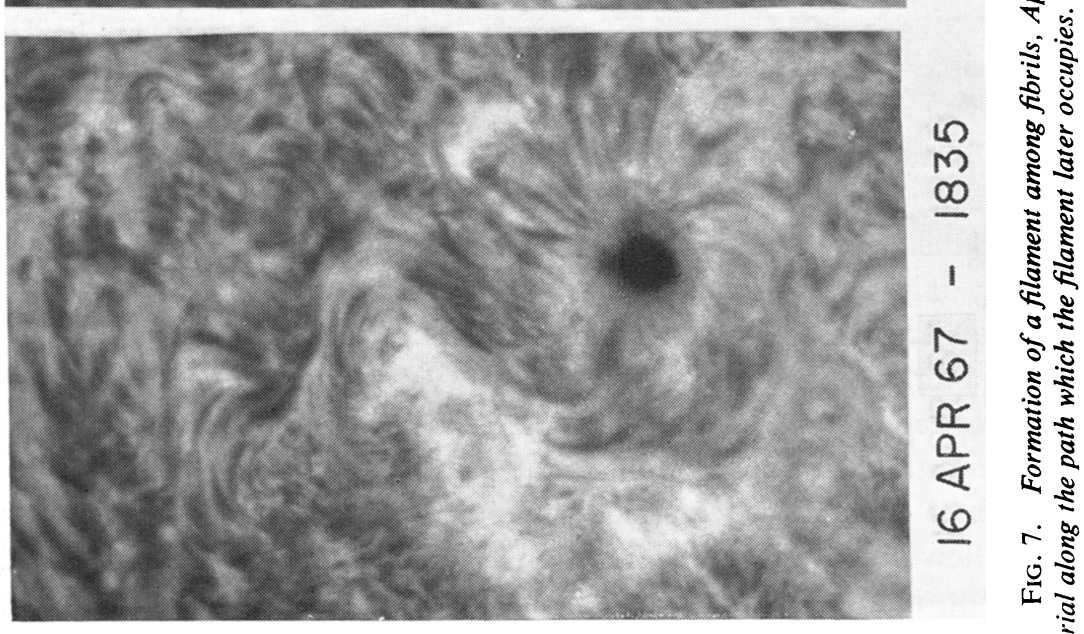

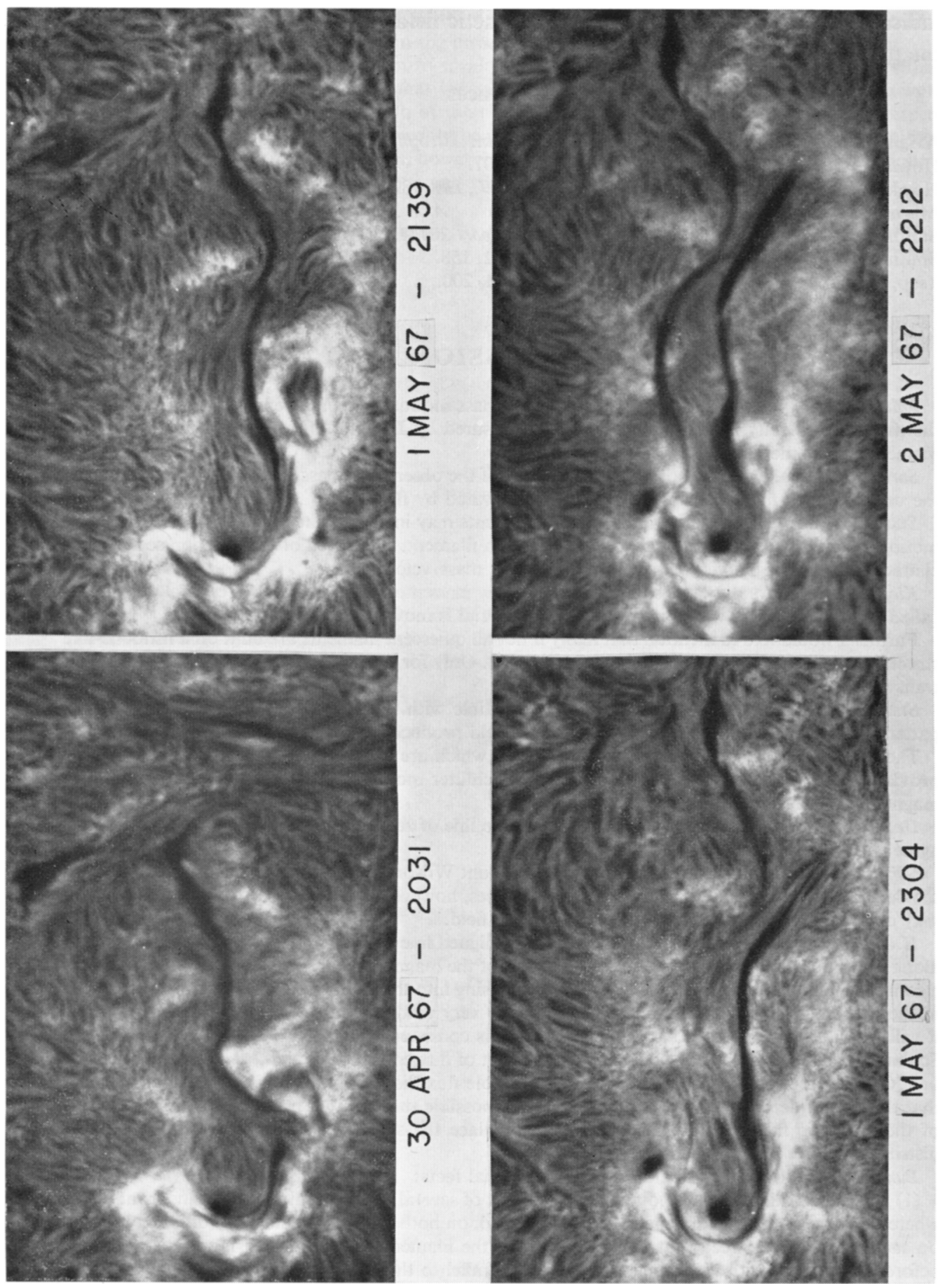

s

궁

:

సे

จ

\&

รี

ำ

รัป

$\stackrel{5}{2.5}$

ป ะ

ปั

ป

帘

ริำ

ब

₹

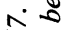

ำ

iั

ฐ

- ذँ

दे हे

จ.ฐ

रे

४ कू

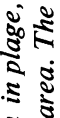

气

5 :

ปั

ริ

ฐ

视

ㅊำ

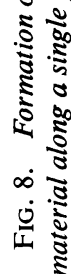


indicates that the lines of force of the magnetic field in filaments, are directed along the path of filaments.

\section{References}

Avignon, Y., Martres, M.J., Pick, M. (1964) Ann. Astrophys., 27, 23.

Howard, R.F., (1959) Astrophys. J., 130, 193-201.

Howard, R.F., Harvey, J. W. (1964) Astrophys. J., 139, 1328-35.

Martres, M.J., Michard, R., Soru-Iscovici, I. (1966) Ann. Astrophys., 29, 245.

Smith, S. F., Ramsey, H.E. (1966) Lockheed Report 20444.

Smith, S.F., Ramsey, H.E. (1967) Solar Phys., 2, 158.

Tsap, T. (1963) Izvest. Krymsk. astrofiz. Obs., 31, 200.

\section{DISCUSSION}

Falciani: Have you measured the velocity of the mass motion in the filament and, in this case, does the measured velocity agree with the velocity measured by Doppler effect, for example, when the filament is near the limb?

Sara Smith: No, we have not yet made a study of the observable mass motion in filaments during the usual state of filaments, that is, when not activated by flares.

Sturrock: The apparent mass motion along filaments may in fact be the result of vertical up-down motion which propagates as an Alfvén wave along a filament. The speed of propagation seems more characteristic of the Alfvén velocity than of likely mass velocities.

Kiepenheuer: I have difficulties to understand, how mass motion in a filament can follow the socalled neutral line? This would imply, that the material is moving across the field.

Further I would like to mention that many if not all quiescent filaments are built on a fishbone-like structure, slightly inclined to the axis of the filament. Only for active filaments close to sunspots such transverse structures do not seem to occur.

Sturrock: The fine structure seems to be compatible with my suggestion that the magnetic-field pattern associated with a filament is a force-free field produced by slippage at the neutral line.

The fact that the magnetic-field patterns shown, which are associated with filaments, are bipolar provides evidence contradicting the Kippenhahn-Schlüter model, which requires a line-quadrupole magnetic-field pattern at the photosphere.

De Jager: Are filaments always oriented along the line of zero longitudinal field? Does your result agree with that of Ioshpa?

Sara Smith: Yes, within the accuracy of the Mount Wilson magnetograms that we have used for the determination of filament positions. In some cases, however, it may be that the end of a filament may deviate from the neutral line of a longitudinal field.

H.U. Schmidt: It seems to me that motions and aligned fine structure almost along a filament and along the neutral line must be due to a component of the magnetic field in the same direction. If such a component is present in the coronal matter condensing into the filament, it will be strongly enhanced in the process of condensation. Therefore it can be very weak in the beginning, and in many cases it will be due to differential rotation. This concept is consistent with the model of Kippenhahn and Schlüter, since there must still be a sufficient amount of flux crossing the filament overlying a neutral line. On the other hand, the absence of a quadrupole flux distribution below the filament indicates that this model needs some modification. It seems possible that the random walk of the base points of the magnetic flux near the neutral line can replace the stabilizing effect of a quadrupole flux distribution.

Bumba: I should like to mention two observational facts:

(1) The feet of filaments are going from junctions of several supergranules, this means from places where the concentration of magnetic field is observed, on both sides of the filament (different polarity on each side), and the feet joint the main body of the filament with the decreasing angle, going just before the junction with the filament practically parallel to the main body of the filament. 
(2) Not only before the appearance of the filament but also after its disappearance it is possible to observe the dark fibrils elongated in the direction and on the place of the previous filament.

Both these observational facts seem to speak in favour of ideas mentioned in Mrs. Smith's talk. Newkirk: Although it is not clear that the same type of prominence is discussed here, the work of Rust suggests that a major fraction of the magnetic fields in quiescent prominences are perpendicular to the axis of the filament, as would be required by the theory of Kippenhahn and Schlüter. A fairly large component of the field is, however, found perpendicular to the filament axis suggesting that a sheared magnetic configuration is present in quiescent prominences. 